\title{
Kompromisse beim Klimaschutz
}

\author{
Ob Umweltpolitik im politischen Kanon eines Landes Gewicht hat, wird auch \\ durch den Interaktionsstil der einzelnen Akteure im jeweiligen Politikfeld \\ bedingt. Eine Analyse der Akteurskonfiguration im Bereich Klimaschutz zeigt, \\ dass Kooperation und Offenheit zum Erfolg von Klimapolitik beitragen.
}

D eutschland wird i gleich häufig als ein Vorreiter in der Umweltpolitik wahrgenommen, besonders seit der Übernahme der Regierungsverantwortung durch die rotgrüne Koalition im Jahr 1998. Inwieweit ist dies auf die Akteurskonfiguration zurückzuführen? Wir untersuchten diese Frage am Beispiel der Analyse des Politiknetzwerkes, das die deutsche Position in den internationalen Verhandlungen zum Klimaschutz bestimmt (1).

\section{- Kapazität und Kooperation}

Die umweltpolitische Kapazität von Ländern wird maßgeblich durch die Zusammensetzung und die Interaktionen der Akteure in den umweltpolitischen Politiknetzwerken bestimmt. Welche Zugangschancen haben Umweltinteressen, auf welche Ressourcen können sie zurückgreifen und inwieweit werden sie als legitime Partner im politischen Prozess anerkannt? Inwieweit bestimmen Kooperation oder Konfrontation den umweltpolitischen Prozess? Bisherige Studien der umweltpolitischen Performanz von Ländern legen nahe, dass die Effektivität politischer Systeme von (a) der relativen Stärke von Umweltakteuren, (b) dem Grad der Kooperation mit anderen gesellschaftlichen Akteuren und (c) der Integration der verschiedenen Sektoren und Ebenen des politischen Handelns abhängen (2).

Die relative Stärke von Akteuren ergibt sich aus einer Vielfalt von Faktoren wie ökonomischen Ressourcen (Anzahl der Mitglieder, Umsatz, Beschäftigte), institutionellem Einfluss wie etwa durch Vetomöglichkeiten, Wissen und Kompetenz oder Glaubwürdigkeit in der Öffentlichkeit. Die Fähigkeit von Akteuren politische Entscheidungen zu beeinflussen wird von einer Kombination dieser Faktoren bestimmt, die - bis zu einem gewissen Grade untereinander substituiert werden können.

Kooperation bezieht sich auf die Fähigkeit und Bereitschaft der politischen und gesellschaftlichen Akteure in der Problemlösung zu kooperieren. Es kann erwartet werden, dass politische Systeme mit einer kooperativen Kultur effektiver sind als solche mit eher konfliktorientierten Strukturen, weil die Ressourcen der beteiligten Akteure eher in die Problemlösung investiert werden, als in den Konflikt mit anderen und dass die Umsetzungschancen gemeinsam getroffener Entscheidungen höher sind. Politische Systeme unterscheiden sich schließlich hinsichtlich des Grades der Integration oder Fragmentierung der verschiedenen Ressorts und der Ebenen politischen Handelns. Querschnittsthemen wie der Schutz der Umwelt erfordern die Zusammenarbeit unterschiedlicher staatlicher Akteure. Die Kooperation zwischen den staatlichen Akteuren wird durch eine Balance bei der institutionellen Kompetenz der Akteure gefördert.

Aus diesen Überlegungen lässt sich die Hypothese ableiten, dass politische Systeme, die offen für die Einbeziehung von Akteuren und ihren Interessen in die Entscheidungsfindung sind, eine bessere umweltpolitische Performanz aufweisen, als politische Systeme, die einen geringeren Grad an Offenheit aufweisen. Der Grad der Offenheit wird durch institutionelle Faktoren bestimmt, aber auch durch die politische Kultur und durch persönliche Faktoren. Wir haben im Rahmen einer Netzwerkanalyse für die deutsche Klimaschutzpolitik die Akteurskonstellation rekonstruiert um diese Argumente zu überprüfen. Ziel war es für den deutschen Fall die relative Stärke der beteiligten Akteure zu bestimmen, zu untersuchen inwieweit diese untereinander kooperieren und wesentliche Interessen integriert sind. Damit soll ein Beitrag zur Erklärung der deutschen Vorreiterrolle im Klimaschutz geliefert werden.

Im Einzelnen haben wir in Interviews Fragen hinsichtlich der Geschichte und der Ressourcenausstattung der Akteure gestellt, haben nach dem Austausch von Informationen, nach Kooperationen und Konflikten mit den anderen Akteuren gefragt und wir haben gefragt, welcher Einfluss im politischen Entscheidungsprozess anderen Akteuren zugemessen wird.
Die Klimaschutzpolitik betrifft nahezu jeden wirtschaftlichen Sektor in Deutschland, entsprechend die Mehrzahl der politischen Ressorts und zahlreiche Interessengruppen. Um die Untersuchung handhabbar zu halten fokussieren wir auf das Netzwerk, das die deutsche Position in der internationalen Klimaschutzpolitik bestimmt und untersucht, welchen Einfluss diese Akteure auf die Ausgestaltung der Ökosteuer genommen haben. In einem mehrstufigen Verfahren bestimmten wir 92 Akteure, die die wichtigsten Organisationen in der Klimapolitik repräsentieren. Diese lassen sich in vier Kategorien klassifizieren: Staatliche Institutionen (29 Akteure), Forschungseinrichtungen (27 Akteure), NGOs (9 Akteure) und Wirtschaftsverbände, einschließlich Gewerkschaften (27 Akteure). Vertreter von 53 dieser Organisationen nahmen an einem telefonischen Interview teil.

\section{Ressourcen der Akteure}

Neben den institutionellen Partizipationschancen kann erwartet werden, dass die finanzielle und personelle Ausstattung, sowie der Zugang zu Informationen die Möglichkeiten zur Beeinflussung politischer Entscheidungen determiniert. Unsere Erhebungen zeigen, dass unter den befragten Akteuren die NGOs vergleichsweise schlecht ausgestattet sind, obwohl unter diesen große Verbände wie Greenpeace, BUND oder NABU vertreten sind. Sie verfuigen über die geringste Zahl von Beschättigten und die kleinsten Budgets verglichen mit den anderen Akteurstypen. Es wäre aber zu einfach, den politischen Einfluss ausschließlich auf diese Ressourcen zu reduzieren. Vielmehr hat beispielsweise die Debatte um die Einführung der Ökosteuer gezeigt, dass die Kampagnen der Umweltverbände einen erheblichen Einfluss hatten (3).

Expertise, Glaubwürdigkeit und die Kooperation mit staatlichen Akteuren kann das Fehlen von finanziellen und personellen Ressourcen ausgleichen. Diese Einschätzung wird auch von den Befragten selber bestätigt, so nennen 41,5 Prozent eine hohe Qualität des wissenschaftlichen Personals oder 35,8 Prozent gute Beziehungen zu staatlichen Akteuren als wichtige Quellen zur Beeinflussung von Entscheidungen, dagegen nur 15,1 Prozent die Qualität und Quantität des Personals insgesamt oder nur 3,8 Prozent das Budget als Voraussetzung.

\section{- Formen der Einflussnahme}

Wir haben danach gefragt, welche Formen der Einflussnahme im Zusammenhang mit der Einführung der Öko-Steuer gewählt wurden (vgl. Abb. 1). Es 


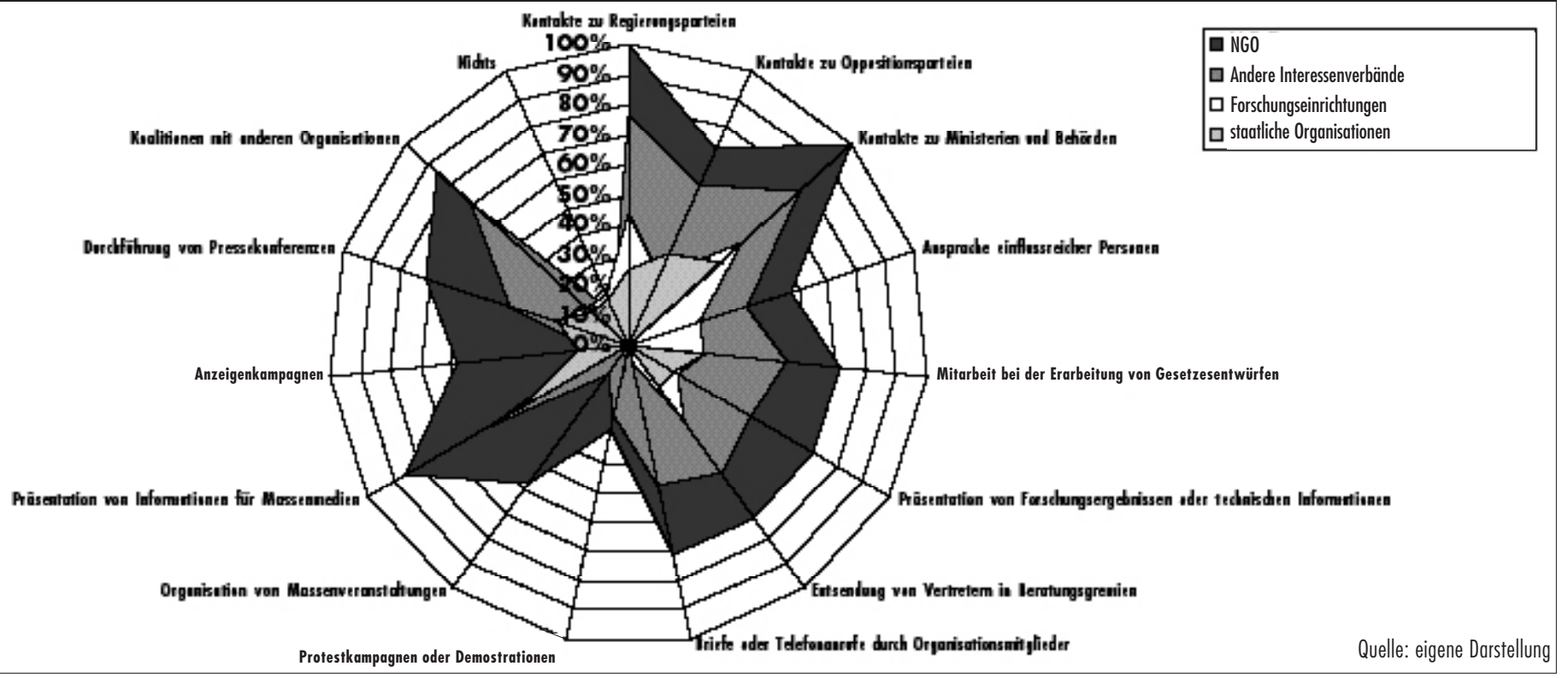

zeigte sich, dass sich alle Akteure mit ihren Aktivitäten vorrangig an staatliche Institutionen richten. Hierzu zählen auch die Mitarbeit bei der Erarbeitung von Gesetzesentwürfen und die Pflege von Kontakten mit der Opposition. Nur die Umweltverbände haben in nennenswertem Umfang auch über die allgemeine Öffentlichkeit oder die Medien versucht, Einfluss zu nehmen. Im Vergleich zu allen anderen Akteurstypen haben sich Umweltverbände auch anderen Formen der Einflussnahme häufiger bedient. Die Mobilisierung der Öffentlichkeit dürfte dazu beitragen, die vergleichsweise kleine Zahl von Mitgliedern zu kompensieren.

\section{- Konflikt und Kooperation}

Die Art und Reichweite von Klimaschutzpolitik ist innerhalb des Netzwerkes Gegenstand kontroverser Auseinandersetzung. Wir haben gefragt, wie Konflikte gelöst und die unterschiedlichen Positionen im politischen Prozess integriert werden. Unsere Erhebungen zeigen, dass Forschungseinrichtungen und staatliche Akteure nur selten als Gegner eingestuft werden. Am häufigsten sind NGOs und wirtschaftliche Interessenverbände in Konflikten involviert. Sie nennen sich gegenseitig am häufigsten als Opponenten.

Interessant ist, dass die relative Konflikthaftigkeit zwischen beiden Akteursgruppen sich nicht auf ihre Einbindung in das Handlungsnetz auswirkt. Auch die Interessenverbände nennen nach staatlichen Institutionen NGOs am häufigsten als Informationsquelle sowie als Koalitionspartner.

Dieser Zusammenhang ist auch bei den anderen Akteuren zu beobachten. Diejenigen Organisationen, die untereinander Informationen austau- schen, bezeichnen sich häufig auch als Koalitionspartner. Die Bedeutung der Umweltverbände als Informanten und Kooperationspartner wird nur noch übertroffen von den staatlichen Akteuren, allen voran die Umweltverwaltungen.

Die zentrale Bedeutung beider Akteure hinsichtlich des Austausches von Informationen und der Kooperation korrespondiert schließlich auch mit ihrem Einfluss im politischen Entscheidungsprozess. Unter den Organisationen, die im Netzwerk die höchste Reputation hinsichtlich ihres Einflusses haben, sind Greenpeace, das Umweltbundesamt, aber auch der Verband der Automobilhersteller und das Wuppertal Institut.

Zusammenfassend kann festgehalten werden, dass das Konfliktniveau und der Grad der Fragmentierung gering ist. Innerhalb des Netzwerkes lässt sich zwar ein Gegensatz zwischen ökonomischen und Umweltinteressen feststellen, der auf Informationsaustausch und Kooperationen jedoch keine Auswirkungen hat. Koalitionen finden zwischen allen Akteursgruppen statt, so beispielsweise zwischen Gewerkschaften, Forschungseinrichtungen und Umweltverbänden beim Lobbying für die Ökosteuer. Umweltverbände sind trotz ihrer vergleichsweise schlechten Ressourcenausstattung ein integrierter und akzeptierter Bestandteil des Politiknetzwerkes; ihre zentrale Position wird wohl auch durch ihre Nähe zu Regierungsorganisationen und ihre Expertise begünstigt.

Gleichzeitig sind auch die Interessengruppen aus der Wirtschaft gut integriert. Insgesamt kann das Netzwerk als inklusiv, kooperativ und offen beschrieben werden. Diese Akteurskonstellation macht zwar immer wieder die Aushandlung von
Kompromissen bei der politischen Entscheidungsfindung notwendig, zugleich kann vermutet werden, dass sie auch die Vorreiterrolle Deutschlands begünstigt. Eine Länder vergleichende Analyse mit Ländern, in denen Umweltverbände schwächer sind oder ein konfrontativer Politikstil vorherrscht, steht allerdings noch aus.

\section{Anmerkungen}

(1) Foljanty-Jost, G./ Jacob, K.: The Climate Change Policy Network in Germany. In: European Environment. 14, 1-15, 2004.

(2) Jänicke, M./ Weidner, H.: Successful Environmental Policy - A Critical Evaluation of 24 Cases. Berlin 1995 sowie Weidner, H./ Jänicke, M. (Eds.): Capacity Building in National Environmental Policy. A Comparative Study of 17 Countries. Berlin 2002.

(3) Reiche, D./ Krebs, C.: Der Einstieg in die ökologische Steuerreform. Aufstieg, Restriktionen und Durchsetzung eines umweltpolitischen Themas. Frankfurt a.M. 1999.

\section{Die Autorlnnen}

Dr. Klaus Jacob ist Forschungsleiter der Forschungsstelle für Umweltpolitik (FFU) an der FU Berlin. Dr. Gesine Foljanty-Jost ist Professorin für Japanologie an der Unversität Wittenberg-Halle. Kontakt: FFU, Ihnestr.22, 14195 Berlin. Tel. 03083855098, E-Mail: jacob@zedat.fu-berlin.de, Martin-Luther-Universität Halle-Wittenberg, Seminar für Japanologie, Brandbergweg 23c, 06099 Halle. Tel. 0345-5524331,

E-Mail: foljanty-jost@japanologie.uni-halle.de 
(c) 20I0 Authors; licensee IÖW and oekom verlag. This is an article distributed under the terms of the Creative Commons Attribution Non-Commercial No Derivates License (http://creativecommons.org/licenses/by-nc-nd/3.o/), which permits unrestricted use, distribution, and reproduction in any medium, provided the original work is properly cited. 